\title{
SINGULAR LOCUS OF A SCHUBERT VARIETY
}

\author{
BY V. LAKSHMIBAI ${ }^{1}$ AND C. S. SESHADRI
}

In this note we give a characterization of the singular locus of a Schubert variety in the flag variety $G / B$, where $G$ is a classical group and $B$ is a Borel subgroup of $G$, or, more generally, for a Schubert variety in $G / Q$, where $Q$ is a parabolic subgroup of $G$ of classical type (cf. $[\mathbf{4}, 5]$ ) of a semisimple algebraic group $G$. This turns out to be a rather easy consequence of the standard monomial theory, developed in Geometry of $G / P, \mathrm{I}-\mathrm{V}$ (cf. $[\mathbf{9}, \mathbf{6}, \mathbf{3}$, $4,7]) .{ }^{1}$ A corollary of this theory is the determination of the ideal defining $X$ in $G / B$, and our result follows from the Jacobian criterion for smoothness. When $G=\mathrm{SL}(n)$ this characterization takes a particularly nice form (cf. Theorem 1 and Remark 1). To the best of our knowledge, our result is new even for a Schubert variety in a Grassmannian. ${ }^{2}$

Let $G$ be a semisimple algebraic group, which we assume, for simplicity, to be defined over an algebraically closed field of characteristic zero (the following discussion is valid in any characteristic, in fact even over $\mathbf{Z}$ ). Let $T$ be a maximal torus, $B$ a Borel subgroup containing $T$, and $W$ the Weyl group of $G$. Let $L$ be a line bundle on $G / B$ associated to a dominant character $\chi$ of $T$ (or $B$ ). Let $V$ be the $G$-module $H^{0}(G / B, L)$. Recall that $V$ can be identified with the set of regular functions $f$ on $G$ such that

$$
f(g b)=\chi(b) f(g) ; \quad b \in B, g \in G .
$$

Now $V$ is also a $\mathfrak{G}$-module, where $\mathfrak{G}=\operatorname{Lie} G$. We identify $Y \in \mathfrak{G}$ with the corresponding right invariant vector field $D_{Y}$ on $G$ and if $v \in V$ corresponds to a function $f$ on $G$ as above, we see that $D_{Y} f=Y f$.

Let $U^{-}$be the unipotent part of the Borel subgroup $B^{-}$, opposite to $B$. We can identify $U^{-}$as an affine open subset (called the opposite big cell) of $G / B$ containing $e_{B} \in G / B\left(e_{B}=\right.$ the point of $G / B$ corresponding to the coset $B$ ). Let $\Delta$ (resp. $\Delta^{+}$) be the set of all (resp. positive) roots. Then recall that

$$
U^{-}=\prod_{\alpha \in \Delta^{+}} U_{-\alpha}, \quad U_{-\alpha} \simeq \mathbf{G}_{a} .
$$

We denote by $\left\{x_{-\alpha}\right\}, \alpha \in \Delta^{+}$, the canonical coordinate system for $U^{-}$given by (1). Now we note that if $f \in V$ as above, and we let $f$ be the restriction of $f$ (identified with a function on $G$ as above) to $U^{-}$, then the evaluations of $\partial f / \partial X_{-\alpha}$ and $X_{-\alpha} f$ at $e_{B}$ coincide.

\footnotetext{
Received by the editors April 13, 1983.

1980 Mathematics Subject Classification. Primary 14M15; Secondary 20 G05.

${ }^{1}$ Partially supported by N.S.F.

${ }^{2}$ In a recent talk at Tata Institute, Bombay, Abhyankar gave a class of singular Schubert varieties in $\operatorname{SL}(n) / B$. 
Let $w \in W$. Then $w$ corresponds to a point $e(w)$ in $G / B\left(e_{B}=e(\mathrm{id})\right)$. The Schubert variety $X(w)$ is, by definition, the Zariski closure of $B e(w)$ in $G / B$ endowed with the canonical reduced structure.

Now let $G$ be a classical group of $\operatorname{rank} l$, and $V_{j}, 1 \leq j \leq l$, the fundamental representation of $G$ with highest weight $i\left(\omega_{j}\right)\left(i=\right.$ Weyl involution and the $\omega_{j}$ are the fundamental weights as in [1]). Then by the first basis theorem of standard monomial theory, $V_{j}$ has a basis $\{p(\lambda, \mu)\}$ of weight vectors indexed by the admissible pairs $(\lambda, \mu)$ in $W / W_{j}\left(W_{j}=W_{P_{j}}, P_{j}\right.$ being the parabolic subgroup associated to $\left.\omega_{j}\right)$, and $G / P_{j}$ is imbedded in $\mathbf{P}\left(V_{j}^{\vee}\right)\left(V_{j}^{\vee}=\right.$ dual of $\left.V_{j}\right)$ (cf. [4, Theorem 5.10]). If the admissible pair is trivial, i.e. $\lambda=\mu$, we write $p(\lambda, \mu)=p(\lambda)$. Recall that:

$$
\left\{\begin{array}{l}
p(\lambda)=\lambda \cdot p(\mathrm{id}) \\
p(\mathrm{id})=\text { lowest weight vector in } V_{j}\left(\text { of weight }-\omega_{j}\right) \\
p(\lambda)(e(\mu)) \neq 0 \text { iff } \lambda \equiv \mu\left(\bmod W_{j}\right) \\
p(\lambda, \mu)(e(\tau))=0 \text { if } \lambda \not \equiv \mu\left(\bmod W_{j}\right) \text { for any } \tau \in W
\end{array}\right.
$$

Let $Y(w)$ denote the intersection of $X(w)$ with the opposite big cell $U^{-}$of $G / B$. Then the principal result we make use of is the following (cf. $[\mathbf{7}, 8]$ ).

LEMMA 1. Let I be the ideal of $Y(w)$ in the coordinate ring of $U^{-}$. Let $w_{j}$ denote the image of $w$ in $W / W_{j}$. Then $I$ is generated by all $p(\lambda, \mu)$ (rather their restrictions to $U^{-}$) such that:

(i) $(\lambda, \mu)$ is an admissible pair in $W / W_{j}(1 \leq j \leq l)$;

(ii) $w_{j} \nsupseteq \lambda$.

PrOOF. This is an immediate consequence of [7, Theorem 10.3], which says that the ideal sheaf of $X(w)$ on $G / B$ is generated by all $p(\lambda, \mu)$ as in (i) and (ii).

Let $I(\omega)$ be the set of all $p(\lambda, \mu)$ as in Lemma 1 . To determine whether $X(w)$ has a singularity at $e_{B}$, we need only determine the rank of the Jacobian matrix $J_{w}$ of $I(w)$ evaluated at $e_{B}$, i.e. the rank of $J_{w}\left(e_{B}\right)$, where

$$
J_{w}=\left\|X_{-\alpha} p(\lambda, \mu)\right\| \quad(\alpha=\text { column index },(\lambda, \mu)=\text { row index }) .
$$

Note that by (2),

$$
\left(X_{-\alpha} p(\lambda, \mu)\right)\left(e_{B}\right) \neq 0 \text { iff } X_{-\alpha} p(\lambda, \mu)=c p(\text { id }) \text { for a nonzero }
$$
scalar $c$.

In particular, if $X_{-\alpha} p(\lambda, \mu) \neq 0$, we see that the weight of $p(\lambda, \mu)=-\omega_{j}+$ $\alpha\left((\lambda, \mu)\right.$ being an admissible pair in $\left.W / W_{j}\right)$. Hence, by weight considerations, we see that in each row of $J_{w}\left(e_{B}\right)$ there is at most one nonzero entry. Now this immediately yields the following:

PROPOSITION 2. The rank of $J_{w}\left(e_{B}\right)=\# R(w)$, where $R(w)$ is the set of all $\alpha \in \Delta^{+}$such that there exists $a(\lambda, \mu) \in I(w)$ with $X_{-\alpha} p(\lambda, \mu)=c p(\mathrm{id})$, $c \neq 0$.

COROLlARY. The dimension of the Zariski tangent space $T_{X(w), e_{B}}$ of $X(w)$ at $e_{B}$ is $N-\# R(w)$, where $N=\# \Delta^{+}$. In particular, $X(w)$ is smooth 
(one knows that $X(w)$ is smooth iff it is smooth at $e_{B}$ ) iff $N-\# R(w)=l(w)$, $l(w)$ being the length of $w$.

Let $\tau \in W$ be such that $w \geq \tau$ so $e(\tau) \in X(w)$. We see that $\left(\tau U^{-} \tau^{-1}\right) \cdot \tau=$ $U_{\tau}^{-} \cdot \tau$ is an affine neighbourhood of $e(\tau)$ in $G / B$. To decide whether $X(w)$ is smooth at $e(\tau)$, it is easily seen that we need only compute the rank $J_{w, \tau}(e(\tau))$, where

$$
J_{w, \tau}=\left\|X_{-\alpha} p(\lambda, \mu)\right\|, \quad \alpha \in \tau\left(\Delta^{+}\right) ;(\lambda, \mu) \in I(w) .
$$

(A better method would be to take the pull-back $\tilde{I}(w)$ of $I(w)$ in $G$ and calculate the rank of the Jacobian matrix of $\tilde{I}(w)$ at a point of $G$ representing $e(\tau)$ using the right invariant vector fields $D_{X_{\alpha}}$ on $G, \alpha \in \Delta$. Observe that $\left(X_{\alpha} p(\lambda, \mu)\right)(e(\tau))=0$ for $\alpha \in \tau\left(\Delta^{+}\right)$.) The above discussion carries over easily and we obtain

Proposition 3. The rank of $J_{w, \tau}(e(\tau))=\# R(w, \tau)$, where $R(w, \tau)$ is the set of all $\alpha \in \tau\left(\Delta^{+}\right)$such that there exists $a(\lambda, \mu) \in I(w)$ with $X_{-\alpha} p(\lambda, \mu)=$ $c p(\tau), c \neq 0$.

Let $G=\mathrm{SL}(l+1)$. Recall that every admissible pair is trivial. We see that if $X_{-\alpha} p(\lambda)$ is nonzero for $\lambda$ in $W$ then

$$
X_{-\alpha} p(\lambda)=p\left(s_{\alpha} \lambda\right) .
$$

Now

$$
\lambda \in I(w) \text { iff } \quad w_{j} \not \lambda \quad \text { in } W / W_{j} \text { for some } j .
$$

Combining (4) and (5) we easily obtain that

$$
X_{-\alpha} p(\lambda)=c p(\tau), c \neq 0 \text {, for some } \lambda \in I(w) \text { iff } w \nsupseteq s_{\alpha} \tau \text { in } W .
$$

Hence we obtain that $R(w, \tau)$ can be canonically identified with the set

$$
R(w, \tau)=\left\{\alpha \in \tau\left(\Delta^{+}\right) \mid w \nsupseteq s_{\alpha} \tau \text { in } W\right\},
$$

and that

(8) $N-\# R(w, \tau)=\# Z(w, \tau)$, where $Z(w, \tau)=\left\{\alpha \in \tau\left(\Delta^{+}\right) \mid w \geq s_{\alpha} \tau\right.$ in $\left.W\right\}$.

Thus we obtain

THEOREM 1. Let $G=\operatorname{SL}(l+1)$. Let $e(\tau) \in X(w)$ with $\tau, w \in W$. Then the dimension of the Zariski tangent space $T_{X(w), e(\tau)}$ of $X(w)$ at e $(\tau)$ is equal to $\# Z(w, \tau)$ so that $X(w)$ is smooth at $e(\tau)$ if and only if $\# Z(w, \tau)=l(w)$. In particular, $X(w)$ is smooth iff

$$
l(w)=\#\left\{\alpha \in \Delta^{+} \mid w \geq s_{\alpha} \text { in } W\right\}
$$

REMARK 1. Using Theorem 1, V. Deodhar has shown that a Schubert variety $X(w)$ in $\mathrm{SL}(l+1) / B$ is smooth at $e(\tau)$ if and only if it is rationally smooth at $e(\tau)$ in the sense of Kazhdan-Lusztig (cf. a forthcoming note of Deodhar).

Let $G$ be any semisimple, simply-connected algebraic group. Let $L_{j}$ be the line bundle on $G / B$ such that $H^{0}\left(G / B, L_{j}\right)$ is the fundamental representation 
$V_{j}$. Note that $H^{0}\left(X(w), L_{j}\right)=V_{j}(w)$ is a $B$-module (in particular a $T$ module), hence we can speak of the multiplicity of a weight in $V_{j}(w)$. For $w, \tau \in W$ with $w \geq \tau$, set

$$
\begin{array}{r}
A(w, \tau)=\left\{\alpha \in \tau\left(\Delta^{+}\right) \mid w \geq s_{\alpha}\right\}, \\
B(w, \tau)=\left\{\alpha \in \tau\left(\Delta^{+}\right) \mid \text {multiplicity of }-\tau\left(\omega_{j}\right)+\alpha \text { in } V_{j}(w)\right. \\
\quad \text { is nonzero for some } j\}, \\
C(w, \tau)=\left\{\alpha \in B(w, \tau) \mid \text { multiplicity of }-\tau\left(\omega_{j}\right)+\alpha \text { in } V_{j}(w)\right. \\
\text { is the same as in } \left.V_{j} \text { for some } j\right\} .
\end{array}
$$

To get a better hold for the dimension of $T_{X(w), e(\tau)}$ (or the rank of $J_{w, \tau}(e(\tau))$ ) than that of Proposition 3, the following can be easily proved:

Proposition 4. With $w, \tau$ as above, we have

$$
\# A(w, \tau) \leq \operatorname{dim} T_{X(w), e(\tau)} \leq \# B(w, \tau) .
$$

In fact, on the basis of many examples (including the case of $G_{2}$, using [2]), we would like to make the following

CONJECTURE. For any $G$ (not necessarily classical),

$$
\operatorname{dim} T_{X(w), e(\tau)}=\# C(w, \tau) .
$$

\section{REFERENCES}

1. N. Bourbaki, Groupes et algèbres de Lie, Chaps. IV, VI, Hermann, Paris, 1968.

2. V. Lakshmibai, Standard monomial theory for $G_{2}$ (preprint).

3. V. Lakshmibai, C. Musili and C. S. Seshadri, Geometry of G/P. III, Proc. Indian Nat. Sci. Acad. Part A 87 (1978), 93-177.

4. __ Geometry of $G / P$. IV, Proc. Indian Nat. Sci. Acad. Part A 88 (1979), 280-362.

5. __ Geometry of $G / P$, Bull. Amer. Math. Soc. (N.S.) 1 (1979), 432-436.

6. V. Lakshmibai and C. S. Seshadri, Geometry of $G / P$. II, Proc. Indian Nat. Sci. Acad. Part A 87 (1978), 1-54.

7. _ Geometry of $G / P, \mathrm{~V}$ (preprint).

8. C. Musili and C. S. Seshadri, Variety of complexes (to appear in the volume dedicated to I. R. Shaferevich on his 60th birthday).

9. C. S. Seshadri, Geometry of G/P. I, C. P. Ramanujan: A tribute, 207, Springer-Verlag (published for the Tata Institute, Bombay), 1978.

Department of Mathematics, University of Michigan, Ann ARbor, MICHIGAN 48109

Department of Mathematics, University of CAlifornia, Los Angeles, CALIFORNIA 90024

TAta Institute of Fundamental Research, Bombay, India 